\title{
POINTWISE ESTIMATES FOR CONVEX POLYNOMIAL APPROXIMATION
}

\author{
D. LEVIATAN
}

\begin{abstract}
For a convex function $f \in C[-1,1]$ we construct a sequence of convex polynomials $p_{n}$ of degree not exceeding $n$ such that $\left|f(x)-p_{n}(x)\right| \leq$ $C \omega_{2}\left(f, \sqrt{1-x^{2}} / n\right),-1 \leq x \leq 1$. If in addition $f$ is monotone it follows that the polynomials are also monotone thus providing simultaneous monotone and convex approximation.
\end{abstract}

1. Introduction and main results. In recent years there has been extensive research on monotone polynomial approximation. Lorentz and Zeller [8], Lorentz [7] and finally DeVore [1] have obtained Jackson type estimates on the rate of uniform approximation of monotone functions by monotone polynomials. Their results can be summarized as follows. For a monotone $f \in C^{k}[-1,1]$ there are monotone polynomials $p_{n}$ of degree not exceeding $n$ such that

$$
\left\|f-p_{n}\right\| \leq C n^{-k} \omega\left(f^{(k)}, n^{-1}\right),
$$

where here and in the sequel $C$ is an absolute constant independent of $f$ and $n$; and $\omega(g, \cdot)$ is the usual modulus of continuity of $g$.

Recently Shvedov $[\mathbf{1 0}]$ has extended these results by showing that for a monotone $f \in C[-1,1]$ there are monotone polynomials $p_{n}$ of degree $\leq n$ such that

$$
\left\|f-p_{n}\right\| \leq C \omega_{2}(f, 1 / n)
$$

where $\omega_{2}(f, \cdot)$ is the second modulus of smoothness of $f$. Moreover, he has proved that one cannot expect (2) to hold with $\omega_{3}$ replacing $\omega_{2}$.

Shvedov $[9,10]$ also discussed the question of convex polynomial approximation to convex functions $f \in C[-1,1]$ showing that there exist convex polynomials $p_{n}$ satisfying (2).

Recently DeVore and $\mathrm{Yu}$ [2] have constructed a sequence of monotone polynomials $p_{n}$ associated with a monotone function $f \in C[-1,1]$ and yielding the following pointwise approximation rate:

$$
\left|f(x)-p_{n}(x)\right| \leq C \omega_{2}\left(f, \sqrt{1-x^{2}} / n\right), \quad-1 \leq x \leq 1 .
$$

Denote $\varphi(x)=\sqrt{1-x^{2}}$ and define

$$
\omega_{2}^{\varphi}(f, \delta)=\sup _{\substack{0 \leq h \leq \delta \\-1 \leq x \leq 1}}\left|\Delta_{h \varphi(x)}^{2} f(x)\right|
$$

where

$$
\Delta_{h \varphi(x)}^{2} f(x)=f(x-h \varphi(x))-2 f(x)+f(x+h \varphi(x))
$$

Received by the editors October 28, 1985.

1980 Mathematics Subject Classification (1985 Revision). Primary 41A10, 41A25, 41A29.

Key words and phrases. Degree of convex polynomial approximation, Jackson-TimanTeljakowskii type estimates, moduli of smoothness, the Peetre kernel.. 
if $x \pm h \varphi(x) \in[-1,1]$ and $=0$ elsewhere. Then the author [6] has shown that the DeVore and Yu polynomials [2] also yield uniform monotone approximation at the rate

$$
\left\|f-p_{n}\right\| \leq C \omega_{2}^{\varphi}(f, 1 / n)
$$

which is an improvement of (1), (2) and even (3) in certain cases and which, although being a uniform estimate, takes into account the behavior of $f$ near the endpoints of the interval allowing rougher behavior of $f$ near the endpoints than in the middle of the interval.

The $\omega_{2}^{\varphi}$ modulus of smoothness has recently been used extensively by Ditzian $[\mathbf{3}, \mathbf{4}]$ and Totik $[\mathbf{1 1}]$ and in a yet unpublished paper by Ditzian and Totik [5]. It is equivalent to the Peetre functional

$$
K_{2}(f, t)=\inf \left\{\|f-g\|_{\infty}+t^{2}\left\|\left(1-x^{2}\right) g^{\prime \prime}(x)\right\|_{\infty}\right\}
$$

where the infimum is taken over all $g \in C^{1}[-1,1]$ such that $g^{\prime}$ is locally absolutely continuous in $[-1,1]$ and $\left(1-x^{2}\right) g^{\prime \prime}(x) \in L_{\infty}[-1,1]$.

In this note we will modify the DeVore-Yu polynomials and for a convex $f$, obtain polynomials which are convex on $[-1,1]$ and which satisfy $(3)$ and (4), thus improving Shvedov's estimates (2). Also, if in addition, $f$ is monotone, then the polynomials are monotone. Our result is therefore

THEOREM 1. There exists an absolute constant $C$ such that for any convex function $f \in C[-1,1]$ and every $n \geq 1$, there is a convex polynomial $p_{n}$ of degree not exceeding $n$ satisfying (3) and (4). If in addition $f$ is monotone, then so is $p_{n}$.

The proof is quite constructive and is the contents of $\S 2$.

Classical converse theorems for algebraic polynomials and Theorem 1 allow us to characterize the convex functions in $\operatorname{Lip}^{*} \alpha, 0<\alpha<2$, by means of their rate of approximation by algebraic polynomials. Since $\operatorname{Lip}^{*} \alpha, 0<\alpha<2$, is defined as the space of all functions $f$ such that $\omega_{2}(f, t)=O\left(t^{\alpha}\right)$, we have

THEOREM 2. For $0<\alpha<2$, a function $f$ is convex and in $\operatorname{Lip}^{*} \alpha$ if and only if for each $n \geq 1$ there is a convex polynomial $p_{n}$ such that

$$
\left|f(x)-p_{n}(x)\right| \leq C\left(\sqrt{1-x^{2}} / n\right)^{\alpha}, \quad-1 \leq x \leq 1 .
$$

2. Proofs. Following [2] we approximate $f$ by a piecewise linear function $S_{n}$ which interpolates $f$ at certain points $-1=\xi_{0}<\xi_{1}<\cdots<\xi_{n}=1$. However, these are fewer points than in [2], in fact, a subset of the points there. The choice of the $\xi_{j}$ 's is made in the following way. Let $J_{n}(t)$ denote the Jackson kernel

$$
J_{n}(t)=\lambda_{n}\left(\frac{\sin n t / 2}{\sin t / 2}\right)^{8}, \quad \int_{-\pi}^{\pi} J_{n}(t) d t=1,
$$

and define

$$
T_{j}(t)=\int_{t-t_{j}}^{t+t_{j}} J_{n}(u) d u, \quad j=0, \ldots, n,
$$

where $t_{j}=j \pi / n, j=0, \ldots, n$. Now for $x=\cos t$ let $r_{j}(x)=T_{n-j}(t)$ and define

$$
R_{j}(x)=\int_{-1}^{x} r_{j}(u) d u, \quad j=0, \ldots, n .
$$


Note that since $T_{0} \equiv 0$ and $T_{n} \equiv 1$ we have $R_{0}(x)=1+x$ and $R_{n}(x) \equiv 0$. The points $\xi_{j}$ are defined by the equations $1-\xi_{j}=R_{j}(1)$.

Like in [2] it follows by the definition of $T_{n}$ that $T_{n-j}-T_{n-(j+1)} \geq 0$. Hence $r_{j}-r_{j+1} \geq 0$ and so $R_{j}-R_{j+1}$ is increasing for $j=0,1, \ldots, n-1$. Therefore $-1=\xi_{0}<\cdots<\xi_{n}=1$. Although our $\xi_{j}$ 's are only a subset of their counterparts in [2] (our $\xi_{j}$ corresponds to $\xi_{2 j-n}, j=0, \ldots, n$ in [2]) they nevertheless have similar distribution in $[-1,1]$. This can be summarized in the following lemma (see [2 or 6]).

LEMmA A. Let $\delta_{j}=\left(\sin t_{n-j}\right) / n+1 / n^{2}, j=0, \ldots, n$. Then

(i) $C_{0} \delta_{j} \leq \xi_{j+1}-\xi_{j} \leq C_{1} \delta_{j}, j=0, \ldots, n-1$,

(ii) $C_{0} \delta_{j} \leq \delta_{j+1} \leq C_{1} \delta_{j}, j=0, \ldots, n-1$,

(iii) for any $\xi_{j} \leq u \leq \xi_{j+1}, 1 \leq j \leq n-2$,

$$
\xi_{j+1}-\xi_{j} \leq C \sqrt{1-u^{2}} / n
$$

Now the piecewise linear interpolant $S_{n}$ in $\left[\xi_{j}, \xi_{j+1}\right]$ has the slope

$$
s_{j}=\frac{f\left(\xi_{j+1}\right)-f\left(\xi_{j}\right)}{\xi_{j+1}-\xi_{j}}, \quad j=0, \ldots, n-1,
$$

and if $\varphi_{j}=\left(x-\xi_{j}\right)_{+}$we can write

$$
S_{n}(x)=f(-1)+s_{0}(1+x)+\sum_{j=1}^{n-1}\left(s_{j}-s_{j-1}\right) \varphi_{j}(x) .
$$

Replacing $\varphi_{j}(x)$ by a sufficiently good approximation to it, namely, $R_{j}(x)$, brings us to the polynomials

$$
\begin{aligned}
L_{n}(f) & =f(-1)+s_{0} R_{0}+\sum_{j=1}^{n-1}\left(s_{j}-s_{j-1}\right) R_{j} \\
& =f(-1)+\sum_{j=0}^{n-1} s_{j}\left(R_{j}-R_{j+1}\right) .
\end{aligned}
$$

It has already been mentioned that $R_{j}-R_{j+1}$ is increasing so that $L_{n}(f)$ is monotone if all $s_{j}$ have the same sign which is the case when $f$ is monotone. Also it follows by Lemma $A$ and the proof in [2] that

$$
\left|f(x)-L_{n}(f)(x)\right| \leq C \omega_{2}\left(f, \sqrt{1-x^{2}} / n\right)
$$

and by the proof in [6] we have

$$
\left\|f-L_{n}(f)\right\| \leq C \omega_{2}^{\varphi}(f, 1 / n) .
$$

Thus our proof is complete if we show that $L_{n}(f)$ is convex when $f$ is. Now when $f$ is convex then $s_{j}-s_{j-1} \geq 0$ for $j=1, \ldots, n-1$; also $R_{0}(x)=1+x$ is linear. Thus it follows by the first equation in (5) that $L_{n}(f)$ is convex if $R_{j}^{\prime \prime} \geq 0$ for 
$j=1, \ldots, n-1$. To prove this we see that for $x=\cos t, 0<t<\pi$,

$$
\begin{aligned}
R_{j}^{\prime \prime}(x)= & r_{j}^{\prime}(x)=\frac{d t}{d x} \frac{d}{d t} \int_{t-t_{n-j}}^{t+t_{n-j}} J_{n}(u) d u \\
= & \frac{-1}{\sin t}\left[J_{n}\left(t+\frac{(n-j) \pi}{n}\right)-J_{n}\left(t-\frac{(n-j) \pi}{n}\right)\right] \\
= & \frac{-\lambda_{n}}{\sin t} \sin ^{8} \frac{n t+(n-j) \pi}{2} \\
& \times\left[\frac{1}{\sin ^{8} \frac{1}{2}(t+(n-j) \pi / n)}-\frac{1}{\sin ^{8} \frac{1}{2}(t-(n-j) \pi / n)}\right] \\
\geq & 0
\end{aligned}
$$

due to the inequality

$$
\sin (\alpha+\beta) \geq|\sin (\alpha-\beta)|, \quad \text { if } 0 \leq \alpha, \beta \leq \pi / 2 .
$$

This was observed by Lorentz and Zeller [8] who used it to obtain estimates on monotone approximation. Surprisingly we apply it to ascertain that the polynomials we have are convex.

\section{REFERENCES}

1. R. A. DeVore, Monotone approximation by polynomials, SIAM J. Math. Anal. 8 (1977), 906-921.

2. R. A. DeVore and X. M. Yu, Pointwise estimates for monotone polynomial approximation, Constr. Approx. 1 (1985), 323-331.

3. Z. Ditzian, On interpolation of $\mathcal{L}_{p}[a, b]$ and weighted Sobolev spaces, Pacific J. Math. 90 (1980), 307-323.

4. __ Polynomials of best approximation in $C[-1,1]$, Israel J. Math. 52 (1985), 341-354.

5. Z. Ditzian and V. Totik, Moduli of smoothness, unpublished manuscript.

6. D. Leviatan, Monotone and comonotone approximation revisited, J. Approx. Theory (to appear).

7. G. G. Lorentz, Monotone approximation, Inequalities. III (O. Shisha, ed.), Academic Press, New York, 1972, pp. 201-215.

8. G. G. Lorentz and K. Zeller, Degree of approximation by monotone polynomials. I, J. Approx. Theory 1 (1968), 501-504.

9. A. S. Shvedov, Jackson's theorem in $\mathcal{L}^{p}, 0<p<1$, for algebraic polynomials and orders of comonotone approximations, Mat. Zametki 25 (1979), 107-117; English transl., Math. Notes 25 (1979), 57-63.

10. (1981), 117-130; English transl., Math. Notes 30 (1981), 63-70.

11. V. Totik, An interpolation theorem and its applications to positive operators, Pacific $\mathrm{J}$. Math. 111 (1984), 447-481.

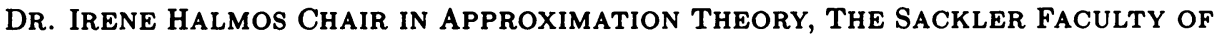
EXACT SCIENCES, TEL-AVIV UNIVERSITY, TEL-AVIV, ISRAEL 78712

DEPARTMENT OF MATHEMATICS, UNIVERSity OF TEXAS AT AUSTIN, Austin, TEXAS 